\title{
Eigenvector centrality for geometric and topological characterization of porous media
}

\author{
Joaquin Jimenez-Martinez* \\ Department of Water Resources and Drinking Water, EAWAG, 8600 Dubendorf, Switzerland; \\ Department of Civil, Environmental and Geomatic Engineering, ETH Zurich, 8093 Zurich, Switzerland; \\ and Earth and Environmental Sciences Division, Los Alamos National Laboratory, Los Alamos, \\ New Mexico 87545, United States \\ Christian F. A. Negre ${ }^{\dagger}$ \\ Theoretical Division, Los Alamos National Laboratory, Los Alamos, New Mexico 87545, United States \\ (Received 19 January 2017; revised manuscript received 16 June 2017; published 13 July 2017)
}

\begin{abstract}
Solving flow and transport through complex geometries such as porous media is computationally difficult. Such calculations usually involve the solution of a system of discretized differential equations, which could lead to extreme computational cost depending on the size of the domain and the accuracy of the model. Geometric simplifications like pore networks, where the pores are represented by nodes and the pore throats by edges connecting pores, have been proposed. These models, despite their ability to preserve the connectivity of the medium, have difficulties capturing preferential paths (high velocity) and stagnation zones (low velocity), as they do not consider the specific relations between nodes. Nonetheless, network theory approaches, where a complex network is a graph, can help to simplify and better understand fluid dynamics and transport in porous media. Here we present an alternative method to address these issues based on eigenvector centrality, which has been corrected to overcome the centralization problem and modified to introduce a bias in the centrality distribution along a particular direction to address the flow and transport anisotropy in porous media. We compare the model predictions with millifluidic transport experiments, which shows that, albeit simple, this technique is computationally efficient and has potential for predicting preferential paths and stagnation zones for flow and transport in porous media. We propose to use the eigenvector centrality probability distribution to compute the entropy as an indicator of the "mixing capacity" of the system.
\end{abstract}

DOI: 10.1103/PhysRevE.96.013310

\section{INTRODUCTION}

A porous medium is composed of interconnected pores (voids or cavities) in a solid material. A fluid (liquid or gas) can flow through a porous medium if the latter is permeable, i.e., if the pores are themselves connected by channels or narrow sections of the pores, usually called pore throats [1], and a potential difference exists. Examples of these systems are common in nature in a wide range of biological (tissues, blood vessels) [2] and inorganic (soil, sedimentary rocks) materials, with long-standing interest for areas such as hydrology (soils and aquifers) [3], petroleum engineering (oil or gas reservoirs) [4], chemical engineering (filters and reactors) [5], and biomechanics [6].

Network models have been used to study fluid flow dynamics and solute transport phenomena in porous media. While early models consisted only of interconnected tubes [7], also called capillary network models [8], more recent models conceptualize the porous medium as a network made up of spherical or polyhedron pores connected by cylindrical (or parallelepipedal) tubes of variable radius and length $[9,10]$. The network that can be extracted from a porous medium, typically only for the connected porosity, is called a pore network. In such a network, there exists a randomness in the spatial distribution, as well as in the weights of the edges, which typically are given a Gaussian distribution with a relatively

\footnotetext{
*joaquin.jimenez@eawag.ch; jjimenez@ethz.ch

†cnegre@lanl.gov
}

small standard deviation. In addition, these types of networks are relatively insensitive to node removal. In other words, for very large networks, several nodes would have to be removed to produce a significant change.

Centrality is a measure of the importance of a node in a complex network with respect to a specific criterion, where several centrality criteria have been proposed. There has been a lot of effort to characterize the centrality of a node in a network (i.e., the relative importance of a particular node), as well as how well connected the network is in general [11,12]. In the case of physical networks it is possible to speak of the centrality of a spatial region of the network and how this will affect network properties. For the particular case of porous media, and for their conceptualization as pore networks, the relation between nodes with respect to flow is important due to the anisotropy: medium properties (e.g., permeability and connectivity-tortuosity, among others) and/or the transfer of a signal through the connected pores depend on the direction of flow. The coexistence of several phases or immiscible fluids in the pore space, besides introducing greater heterogeneity in the system, accentuates the anisotropy. The impact of anisotropy on flow and transport phenomena through porous media is well documented in the literature [13-18]. Therefore, the relative importance (centrality) of a pore (node) in a porous medium (pore network) depends on the mean flow direction.

In this work, we study the geometric and topological properties of a porous medium by using the eigenvector centrality metric (a special measure of node centrality or relative importance) and the impact of the presence of an immiscible fluid phase on it. To the best of our knowledge, this is the 
first time that dynamical properties of fluid flow and solute transport in pore networks are inferred with the eigenvector centrality metric. We propose three methodological novelties: a correction of the eigenvector centrality measure to avoid the problem of artificial centrality localization, a direction-biased adjacency matrix to account for preferential direction affecting node centrality, and a spatially projected eigenvector centrality function for better visualization. Based on this analysis, we infer properties such as the connectivity, percolation threshold, and potential of the porous material for fluid flow and solute transport. Computing the centrality of a region of this physical network provides a powerful tool for predicting the regions (fraction) of the porous medium that behave as preferential paths (regions of high fluid velocity) or stagnation zones (regions of low fluid velocity). In other words, in the case of solute transport, we could infer, within the limitations of the technique, the probability of having fingering [19].

\section{METHODS}

\section{A. Eigenvector centrality}

The pore networks herein considered can be represented as a weighted graph. In the latter, a set $G=(V, E)$ of nodes in $V$ are connected by edges in $E$. Each edge $e_{i j}$ connects nodes $v_{i}$ and $v_{j}$ with 'strength' $w_{i j}$, where $v_{i}$ and $v_{j} \in V$ and $e_{i j} \in E$. Every weighted graph has an associated adjacency matrix defined as

$$
A_{i j}= \begin{cases}0 & \text { if } i=j \\ w_{i j} & \text { if } i \neq j\end{cases}
$$

where $A_{i j}$ is a symmetric $N \times N$ matrix, where $N$ is the total number of nodes. In order to determine which are the most important nodes, many centrality measures have been proposed. One of them, the node degree, consists of simply counting how many neighbors a node has. For the general case of a weighted graph, this is $\operatorname{deg}_{i}=\sum_{k=1}^{N} A_{i k}$. Within this definition, a node will be central if it has "lots of tightly" connected neighbors. The problem with this simple metric lies in that it is a local property and does not take into account the influence of the other nodes in the network. This problem is solved by using the eigenvector centrality measure. Eigenvector centrality, $c$, states that the importance of a node in the network is directly proportional to the importance of its neighbors. Mathematically this is

$$
c_{i}=\frac{1}{\lambda} \sum_{j=1}^{N} A_{i j} c_{j}
$$

with $1 / \lambda$ a proportionality constant. If we reorder Eq. (2) and express it in matrix form, we obtain an eigenvalue equation $A \mathbf{c}=\lambda \mathbf{c}$, with $\lambda$ and $\mathbf{c}$ being the eigenvalue and eigenvector, respectively. If we add the constraint that every component $c_{i}$ cannot be negative, the Perron-Frobenius theorem ensures that, given that $A$ is a symmetric matrix, the eigenvector where $c_{i}>0 \forall i$ is given by the one corresponding to the largest eigenvalue [20].

It is well known that the eigenvector centrality has problems of localization, and a solution to this problem has been proposed recently [21]. Localization occurs when network connectivity is low or when the average degree takes low values (in the case of an unweighted graph, these low values could be close to 1 , meaning only one neighbor). When localization is removed, the principal eigenvalue of the adjacency matrix becomes increasingly dominant, separating from the rest of the eigenvalues (see Appendix A). For sparsely connected networks like the pore networks analyzed here, we found that the principal eigenvalue is close to the secondary eigenvalue, and the result is a localization that remains far from the logical intuition derived from the physical properties of the network (see Appendix A). Finally, for well-connected networks, the localization is lower and the eigenvector centrality yields a reasonable measure.

For regular networks in general, this localization occurs only if there is a degree of randomness in the weights. For example, localization occurs (see Appendix A) if we have a network represented by the following adjacency matrix:

$$
A_{i j}= \begin{cases}0 & \text { if } i=j, \\ \operatorname{rnd}(0,1) & \text { if } i \text { is a neighbor of } j,\end{cases}
$$

where $\operatorname{rnd}(0,1)$ is a random number between 0 and 1 . Localization is removed if $w_{i j}=1$ and if $i$ is a neighbor of $j$ (the case of a lattice). This phenomenon is reminiscent of the "Anderson localization" occurring in quantum electron transport [22].

In order to solve this problem, and to analyze the physical networks introduced here, we have applied an ad hoc correction to avoid the localization, which proves to work remarkably well. We propose adding a spurious value to the adjacency matrix so that

$$
A_{i j}= \begin{cases}0 & \text { if } i=j, \\ w_{i j}+\delta & \text { if } i \neq j,\end{cases}
$$

where the parameter $\delta$ can be chosen between 0 (returning the original matrix) and $\delta=\min _{i j}\left(A_{i j}\right) \forall w_{i j}>0$. Results demonstrate that we can transition from very localized to delocalized centrality values, for low and high values of $\delta$, respectively (see Appendix B). This transition can be viewed as a sort of "contrast" tuning where the relative importance of the nodes can be controlled as desired. For the extreme case where every node is explicitly connected together (choosing $\delta$ such that $A_{i j}=1 \forall i \neq j$ ), there is a dominant eigenvalue for which the eigenvector is $\mathbf{c}=\frac{1}{\sqrt{N}}(1,1, \ldots, 1)$, meaning that all the nodes will have the same relative importance.

The solution herein proposed is equivalent to solutions proposed for other purposes (e.g., the PageRank metric used to measure Web-page rank [23-25]). In these alternative solutions there is a source of centrality artificially introduced and the eigenvalue equation is rewritten as $\left(A+\mathbf{s} \mathbf{1}^{t}\right) \mathbf{c}=\lambda \mathbf{c}$, where $\mathbf{s} \mathbf{1}^{t}$ is the outer product between $\mathbf{s}=(\delta, \ldots, \delta)$ and $\mathbf{1}=(1, \ldots, 1)$. Our correction, instead of a random source of values (i.e., rank), can be seen as a nonzero probability of a pore having a connection to all the nonneighboring pores. In other words, for an uncompressible fluid (e.g., water) in a nondeformable porous medium, a pressure change will be transferred to all the connected pores, or for a solute, it will reach all the connected pores by long-term molecular diffusion. The latter would constitute a plausible physical interpretation to justify the "spurious" connectivity parameter $\delta$. In the case of a spread distribution of weights, the approach 
herein introduced could probably fail, unless small weights are explicitly not considered.

\section{B. Pore-network construction}

The most efficient method for conceptualizing a porous medium is by the pore skeleton or pore network. The original porous medium used in this work comes from experimental results [26] of solute transport in variably saturated conditions (i.e., the presence of two immiscible fluids, water and air). The two-dimensional (2D) porous medium (length $\times$ width $\times$ height $=132 \times 87 \times 0.5 \mathrm{~mm}$ ) consists of a water-wet monolayer of 4421 cylindrical grains. An image of such a system contains $3289 \times 2181$ pixels, corresponding to a spatial resolution of $\simeq 0.04 \mathrm{~mm}$ per pixel $(\simeq 25$ pixels per millimeter). The average grain radius is $r=0.415 \pm 0.11 \mathrm{~mm}$, the average pore size $\varepsilon=1.85 \mathrm{~mm}$, the porosity $\phi=0.71$, and the absolute permeability $\kappa=7.38 \times 10^{-3} \mathrm{~mm}^{2}$. Binary images, with zero at the porous space and one at the grains and the non-wetting phase (air), are used for the pore-network construction [Fig. 1(a)].

For each discretized point of the porous medium (pixel of the image), we assign a number corresponding to its distance from the nearest nonzero point in the porous medium. This leads to a function (Euclidean distance transform [27]) that maps each discretized point to the "distance to nearest objects" (DNO) as shown in Fig. 1(b). For this purpose, we have used the MATLAB routine bwdist [28]. The objects or solid regions of the porous medium include grains and/or nonwetting phase clusters. These solid regions will hence have a DNO value of 0.0 in pixel units [deep-blue areas in Fig. 1(b)].

The network is created by taking the local maxima of the DNO function. Edges are determined with the maxima of the DNO function and node positions are established at the intersection of the edges (the centroid of each pore). Note that films or corners of the wetting phase around isolated grains or grains in contact with the nonwetting phase are not considered in the network construction (see Fig. 1). The resulting network

(a)

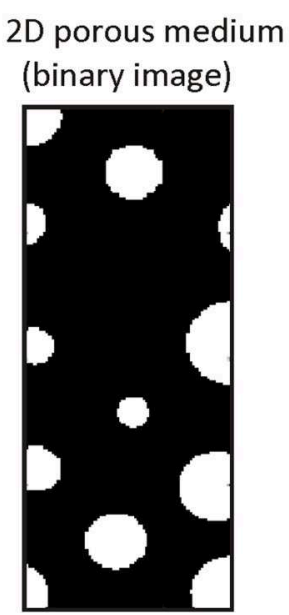

(b)

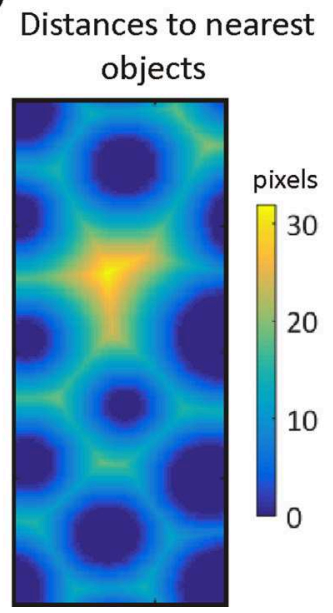

TABLE I. Pore-network statistics for the four saturation degrees $S_{\mathrm{w}}$ investigated: number of dead-end nodes $N_{\mathrm{d}-\mathrm{e}}$, total number of nodes $N$, average pore throat size $\bar{s}$, average distance between nodes or edge length $\bar{d}_{N}$, average degree or number of edges around a node $\overline{\mathrm{deg}}$, average weight or "strength" $\bar{w}$, and maximum eigenvalue $\lambda_{N}$.

\begin{tabular}{cccccccc}
\hline \hline$S_{\mathrm{w}}$ & $N_{\mathrm{d}-\mathrm{e}}$ & $N$ & $\bar{s}$ & $\bar{d}_{N}$ & $\overline{\operatorname{deg}}$ & $\bar{w}$ & $\lambda_{N}$ \\
\hline 1.00 & 282 & 8074 & 20.62 & 26.85 & 2.90 & 0.94 & 10.85 \\
0.83 & 5108 & 12582 & 16.36 & 18.77 & 2.16 & 0.92 & 14.65 \\
0.77 & 5318 & 12267 & 15.70 & 18.31 & 2.11 & 0.92 & 14.28 \\
0.71 & 6484 & 13237 & 14.63 & 16.36 & 1.99 & 0.91 & 15.12 \\
\hline \hline
\end{tabular}

consists of medial axes (edges) converging in the center of the voids or pores (nodes) (e.g., [29,30]). Thus, the pore network can be parameterized by the distance $d$ between nodes (length of the edges) and the cross section of the pore throat $s_{i j}$ normal to the edge (pore-throat size) connecting node $i$ with node $j$ [indicated by the white double-arrow in Fig. 1(c)]. In order to determine $s_{i j}$, we have taken the absolute minimum of the DNO function evaluated along the medial axis that connects the two neighboring pores $i$ and $j$. Similar procedures have been used to compute other geometrical properties of the porous media such as tortuosity and connectivity or to infer the permeability [31]. The pore network constructed in this way could be viewed as a "fingerprint" of the porous medium and the main characteristics of these networks for the different degrees of saturation are listed in Table I.

\section{Adjacency matrix of a pore network}

In order to account for the edge weights between neighboring nodes, we have considered the function

$$
w_{i j}=\exp \left(-s_{0} / s_{i j}\right),
$$

where $s_{i j}$ is the two-dimensional effective cross section (pore throat) that connects node $i$ with node $j . s_{0}$ is a normalization coefficient chosen as 1 pixel unit. By this definition, if pores

(c)

Pore-network

(edges and nodes)

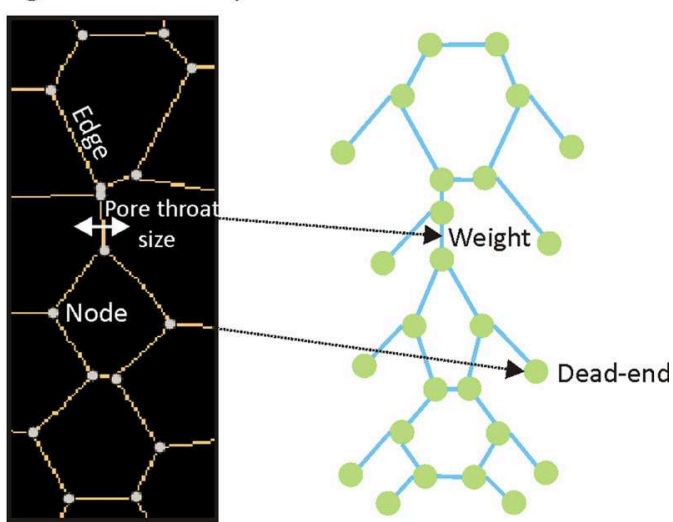

FIG. 1. (a) Binary image of the 2D porous medium, with pores or voids in black and grains in white. (b) Distances to nearest objects (DNO function) inside the pore space. (c) Pore network composed of edges connecting nodes (centroid of the pore) determined by the local maxima of the DNO function. (d) Corresponding graph for the defined pore network. The minimum distance normal to the edge (i.e., pore-throat size) provides the weight. The termination of a path, both in the pore network and in the graph, is called a dead-end. 
$i$ and $j$ are connected by an extremely large pore throat, then $w_{i j} \rightarrow 1.0$, and on the contrary, if the pore throat section is small, then $w_{i j} \rightarrow 0.0$. Moreover, the weights $w_{i j}$ as defined here could be viewed as the probability that a particle or differential volume-mass will flow from pore $i$ to pore $j$ (see Appendix C).

Based on this weight definition, the adjacency matrix can be constructed as follows:

$$
A_{i j}= \begin{cases}0 & \text { if } i=j, \\ \exp \left(-s_{0} / s_{i j}\right) & \text { if } i \text { is a neighbor of } j .\end{cases}
$$

In order to compute the eigenvector centrality, the adjacency matrix was diagonalized after applying Eq. (4) to correct for localization. We have employed the Basic Matrix Library to perform all the linear algebra operations in this work [32]. In order to account for the centrality defined at any point $\mathbf{r}$ of the pore network, we have computed a spatially projected eigenvector centrality (SPEC) function $c(\mathbf{r})$, which we define as

$$
c(\mathbf{r})=\frac{1}{\rho(\mathbf{r})} \sum_{i}^{N} c_{i} \mathcal{L}\left(\mathbf{r}-\mathbf{r}_{i}\right),
$$

where $c_{i}$ and $\mathbf{r}_{i}$ are, respectively, the centrality and position of node $i, \rho(\mathbf{r})$ is the node density at point $\mathbf{r}$, and $\mathcal{L}$ is a $2 \mathrm{D}$ Lorentzian function defined as

$$
\mathcal{L}\left(\mathbf{r}-\mathbf{r}_{i}\right)=\frac{\zeta}{2 \pi} \frac{\Gamma}{\left\|\mathbf{r}-\mathbf{r}_{i}\right\|^{2}+(\Gamma / 2)^{2}},
$$

where $\Gamma$ is a parameter that controls the width of the Lorentzian function. This Lorentzian function is normalized for a restricted domain with a normalization coefficient $\zeta$ to ensure convergence over the network region $D$ (see Appendix D) [33]. The node density $\rho$ is obtained as follows:

$$
\rho(\mathbf{r})=\sum_{i}^{N} \mathcal{L}\left(\mathbf{r}-\mathbf{r}_{i}\right) .
$$

Note that by integrating the node density over the entire network region, we get the total number of nodes, $\iint_{\mathrm{D}} \rho(\mathbf{r}) d s=$ $N$, and $\rho(\mathbf{r}) / N$ can be viewed as the probability density distribution $p(\mathbf{r})$ of finding a node at point $\mathbf{r}$ (see Appendix E). The SPEC function $c(\mathbf{r})$ allows the interpolation of a centrality value to any point $\mathbf{r}$ in the $2 \mathrm{D}$ space of the sample from all the centrality values $c_{i}$ of nodes located at position $\mathbf{r}_{i}$.

\section{Eigenvector centrality conditioned by the mean flow direction: Anisotropy}

In order to account for a conditioned centrality with a preferential direction, due to the anisotropy in porous media, we have modified the weights of the adjacency matrix as

$$
w_{i j}^{(\hat{\mathbf{F}})}=\left|\cos \left(\alpha_{i j}\right)\right| \exp \left(-s_{0} / s_{i j}\right),
$$

where $\cos \left(\alpha_{i j}\right)$ is the director cosine between the direction $\hat{\mathbf{F}}$ and the vector $\mathbf{r}_{i}-\mathbf{r}_{j}$ defined as follows:

$$
\cos \left(\alpha_{i j}\right)=\frac{\left(\mathbf{r}_{i}-\mathbf{r}_{j}\right) \cdot \hat{\mathbf{F}}}{\left\|\mathbf{r}_{i}-\mathbf{r}_{j}\right\|} .
$$

For the cases analyzed here, the mean flow direction will be $\hat{\mathbf{F}}=(1,0)=\hat{x}($ see Appendix F).

\section{RESULTS AND DISCUSSION}

\section{A. Comparison with transport experiments}

Figure 2 shows the results of 2D millifluidic solute transport experiments where the area of a wetting phase (water) reached by a tracer (fluorescein) over the duration of the experiment is determined for both full and partial water saturation conditions $S_{\mathrm{w}}$ (defined as the ratio of the combined area of water clusters with respect to the porous space). Note that in the present case, we do not have multiphase flow [34]: air is introduced to add heterogeneity to the system but does not move during the transport experiments. The injection of the solute tracer in the partially saturated experiments was performed at the same flow rate $\left(Q=0.277 \mathrm{~mm}^{3} \mathrm{~s}^{-1}\right)$, while in the fully saturated experiment (without air) it was injected at a higher flow rate $(Q=$ $\left.1.375 \mathrm{~mm}^{3} \mathrm{~s}^{-1}\right)$. For a detailed description of the experimental setup, protocol, and discussion of transport processes, including solute dispersion and mixing, the reader is referred to [26].

In Fig. 2 we can observe that the tracer only reaches the entire region in the fully saturated case. For the partially saturated cases, a large area (shown in blue) remains unreached by the tracer. This phenomenon is explained by the fact that the heterogeneity introduced by the presence of a nonwetting phase (air) creates regions of the wetting phase with high velocities or preferential paths and regions of low velocities, also called stagnation zones or dead-ends [35]. This more heterogeneous velocity field is responsible for the enhancement of solute dispersion and mixing in unsaturated porous media and, as a consequence, the enhancement of potential chemical reactions $[26,36]$.

The SPEC function conditioned by the mean flow direction ( $x$ direction) is shown as a 2D map for each experimental porous media system in Fig. 2 [26], and a dotted-type plot (showing centrality as a dot in every node) is presented for comparison in Appendix G. This representation allows a visual analysis of the relative importance of a node or region in the pore network, defined for the wetting phase (i.e., excluding clusters of nonwetting phase), from the measure of the eigenvector centrality, by highlighting the preferential paths (high eigenvector centrality values and relative importance for flow, warm colors) and stagnation zones (low eigenvector centrality values and relative importance for flow, cold colors) in a porous medium.

The $x$-biased SPEC function shows a lower relative importance, i.e., lower connectivity, for nodes that are closer to the porous medium borders [Fig. 2(a)]. Note that, despite the fact that the SPEC function is an intrinsic characteristic of the network, the lower values observed at the top and bottom borders in Fig. 2(a) imply a drag for the tracer flux [26]. The latter could be seen as Taylor dispersion [37], which is manifested through a paraboliclike profile of the fluid velocity between two solid-immobile objects, with the highest velocity in the center and lower velocities closer to the objects, being 0 at their surfaces.

Boundary effects are also observed under partially saturated conditions, including around the nonwetting clusters (air), where dead-end nodes are created. The number (and proportion) of dead-ends increases as saturation decreases (Table I). A slip boundary condition is usually considered for fluid flow around air bubbles in multiphase systems, meaning no drag for fluid flow and solute transport. However, the alternation 
(a)

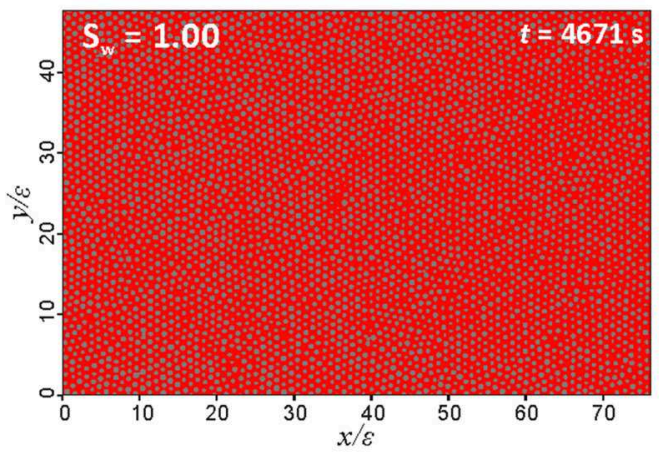

(b)

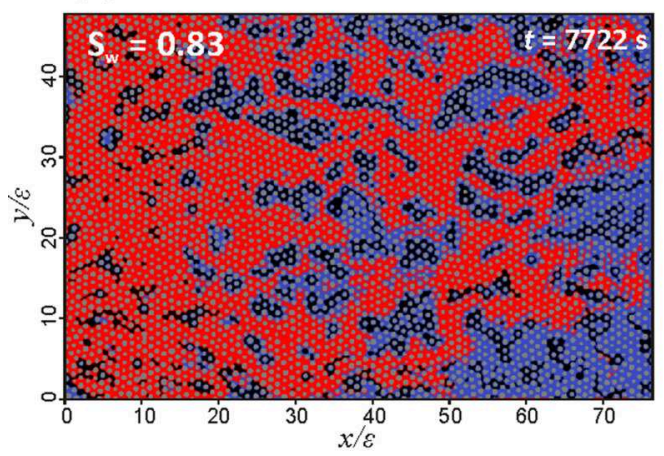

(c)

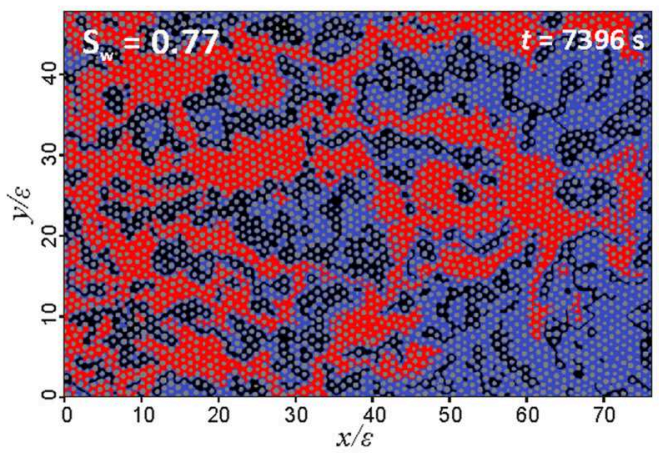

(d)

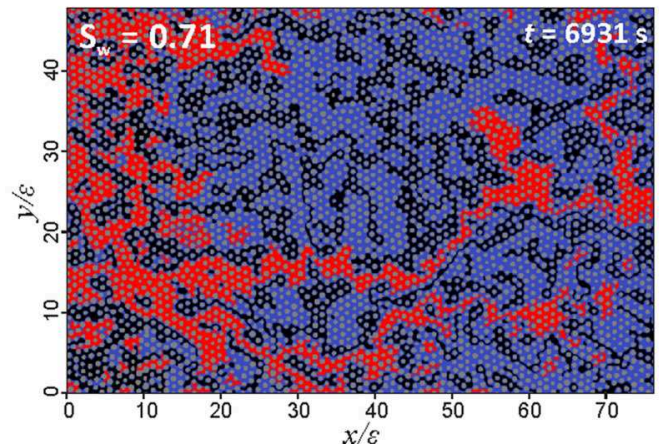

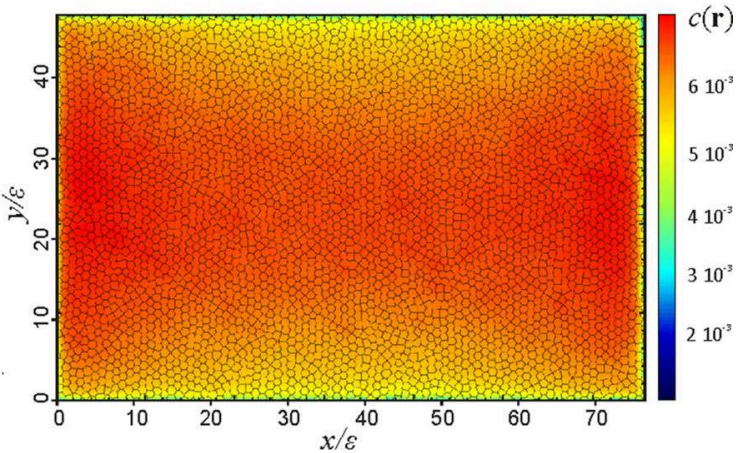
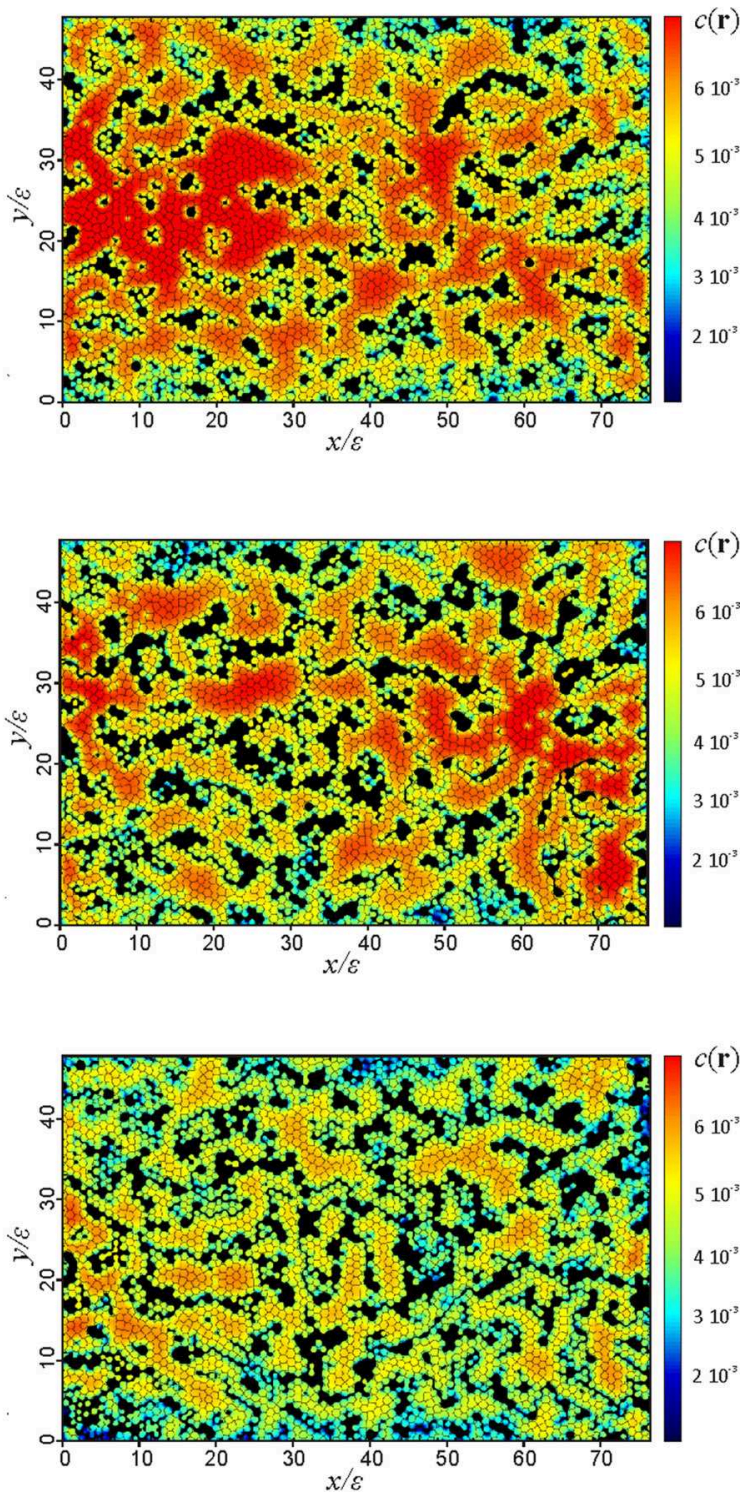

FIG. 2. Left: Millifluidic transport experiments under variably saturated conditions [26]. Area of the wetting phase reached by the tracer (flowing from left to right; entering across the entire $y / \varepsilon$ width at $x / \varepsilon=0$ ) over the duration of the experiment (red areas), for different water saturation $S_{\mathrm{w}}$ values: (a) 1.00 , (b) 0.83 , (c) 0.77 , and (d) 0.71 . Areas without tracer present are shown in blue. Grains are depicted in gray, and air clusters in black. Right: Two-dimensional maps showing the $x$-biased SPEC function $[c(\mathbf{r})]$ for each water saturation value. Warm colors (red and yellow) represent nodes and regions of the pore network with high eigenvector centrality values, i.e., high relative importance for flow and transport. Cold colors (green and blue) represent nodes and regions of the wetting phase with low eigenvector centrality values, i.e., low relative importance in the network. The pore network, including edges and nodes, defined for the wetting phase is plotted with black lines. 


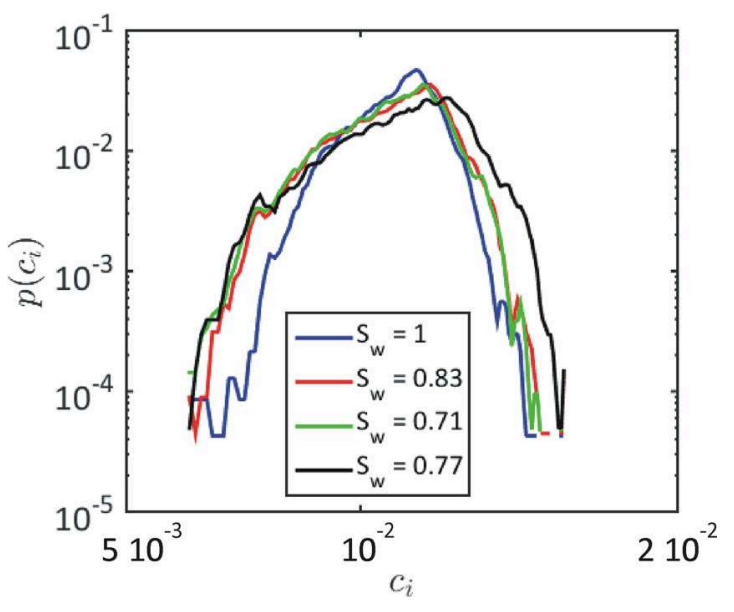

FIG. 3. Eigenvector centrality $\left(c_{i}\right)$ probability density functions for different water saturation values $S_{\mathrm{w}}$.

in the edges of a water cluster of solid grains and water-air interfaces (menisci) results in higher velocities at the center of the water cluster than at the borders, creating a similar solute dispersion to that observed in a channel with a periodically varying aperture [38,39].

\section{B. Impact of saturation on the eigenvector centrality}

The degree of wetting phase saturation $S_{\mathrm{w}}$ has a direct impact on the structure of the pore network. "Desaturation" (water drainage) of the porous medium is carried out to achieve the desired $S_{\mathrm{w}}$ value for solute transport experiments. During this process, capillary forces favor the drainage of large pores, which are occupied by the nonwetting phase (air), and the displacement of the wetting phase (water) across smaller pores (or pores with small entry throats). Computed statistics from the pore networks (Table I) show how the average pore throat size $\bar{s}$ and the average distance between nodes (edge length) $\bar{d}_{N}$ decrease as water saturation decreases. This confirms the drainage from large to smaller pores as $S_{\mathrm{w}}$ decreases. The impact of desaturation on the pore network is also reflected in the average degree or number of edges around a node $\overline{\mathrm{deg}}$, average weight or strength $\bar{w}$, and connectivity (see Table I), discussed below.

We studied the impact of the wetting phase saturation $S_{\mathrm{w}}$ on eigenvector centrality values $c_{i}$ by computing their probability density function (Fig. 3) and the first three moments (Table II). In the probability density functions, the peak in the $p\left(c_{i}\right)$ shifts to a slightly higher value, and the width of the distribution increases, with decreasing $S_{\mathrm{w}}$. As expected,

TABLE II. Moments of the eigenvector centrality distribution, including the mean $\mu_{c_{i}}$, variance $\sigma_{c_{i}}^{2}$, and skewness $\gamma_{c_{i}}$, for different water saturation $S_{\mathrm{w}}$ values.

\begin{tabular}{cccc}
\hline \hline$S_{\mathrm{w}}$ & $\mu_{c_{i}}$ & $\sigma_{c_{i}}^{2}$ & $\gamma_{c_{i}}$ \\
\hline 1.00 & $1.12 \times 10^{-2}$ & $8.21 \times 10^{-7}$ & -0.31 \\
0.83 & $9.30 \times 10^{-3}$ & $5.30 \times 10^{-7}$ & -0.32 \\
0.77 & $9.50 \times 10^{-3}$ & $5.80 \times 10^{-7}$ & -0.30 \\
0.71 & $9.10 \times 10^{-4}$ & $5.29 \times 10^{-7}$ & -0.26 \\
\hline \hline
\end{tabular}

increasing the degree of heterogeneity as $S_{\mathrm{w}}$ decreases broadens the distribution of eigenvector centrality. In the fully saturated example [see Fig. 2(a)], there is a similar spatial eigenvector centrality value across the pore network. However, desaturation creates a more heterogeneous system in which nodes and regions with high and low eigenvector centrality values coexist [see Figs. 2(b)-2(d)].

The first moment, mean $\left(\mu_{c_{i}}\right)$, in general, decreases as $S_{\mathrm{W}}$ decreases. This is explained by the creation of regions with low eigenvector centrality values (stagnation zones) as the saturation decreases. The variance $\left(\sigma_{c_{i}}^{2}\right)$ is similar in the partially saturated experiments, despite the different $\mu_{c_{i}}$. The third moment of the spatial eigenvector centrality, its skewness $\left(\gamma_{c_{i}}\right)$, measures the symmetry of the distribution. In this particular case, a negative $\gamma_{c_{i}}$ indicates the dominance of regions with a low eigenvector centrality value. An analysis of the differences in these parameters is reported in Appendix $\mathrm{H}$.

The fraction $\xi$ of the wetting phase of very low relative importance (low eigenvector centrality values) on solute transport was evaluated by establishing a threshold in $c(\mathbf{r})$. In [26], the fraction of the wetting phase considered not to be involved in the transport process over the total duration of the experiments was determined by establishing a threshold value of 0.01 for the tracer concentration $C$. With this threshold, the full stagnation region is defined by points $(x, y)$, for which $C(x, y)<$ 0.01 . Results in [26] show that the lower the wetting saturation, the larger the area of stagnation zones (low velocities), as shown by the solid line in Fig. 4. This implies that fluid flow and solute transport remain constrained in narrower connected preferential paths for lower values of saturation (Fig. 2).

The trends determined in [26] can also be recovered for a range of centrality thresholds as shown by the shaded area in Fig. 4. Although the trend decreases monotonically for both experiments and calculations, the stagnation-zone fraction $\xi$ tends to diverge for lower saturation values. This can be explained by the fact that the SPEC function ultimately represents a situation where steady state is achieved, whereas experiments are limited by the total execution time. With limited execution time, very well-defined preferential paths are more evident in the experimental case. We can see that preferential paths observed in the experiments fall within the regions depicted by the SPEC function [Figs. 2 and 4(a)].

\section{Entropy, connectivity, and percolation}

Eigenvector centrality as computed in this work can be viewed as a dynamical fingerprint, for both fluid flow and solute transport, of a porous medium or the phases contained within it. Eigenvector centrality measures can also be used to quantify the entropy or disorder and the connectivity of the system given a particular flow direction. Pore-network statistics computed to get the SPEC function can be used to deduce relevant quantities for transport through a porous medium, e.g., the percolation threshold.

Entropy is defined as the degree of disorder or randomness in a system. Different measures of entropy, depending on the purpose, have been proposed [40]. Among them, Shannon entropy, initially proposed for information theory, has been demonstrated to have relevance in other areas such as statistical mechanics [41]. We propose the use of this definition of 
(a)

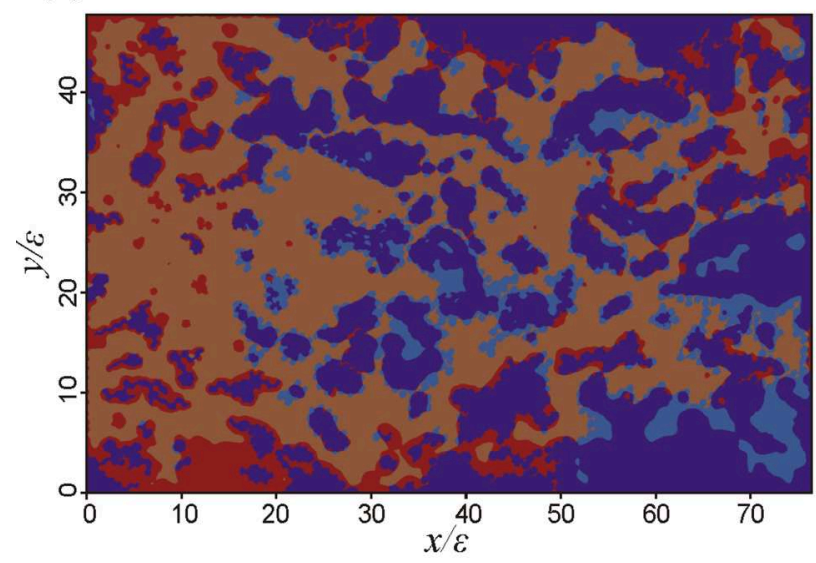

(b)

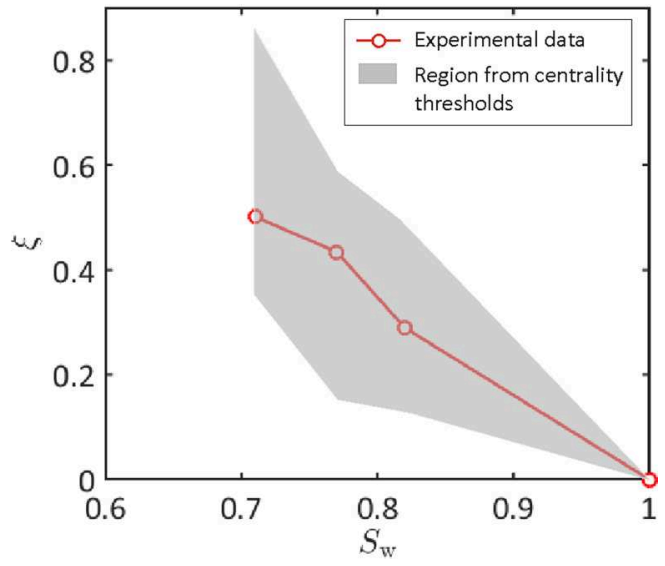

FIG. 4. (a) Transport experiment and SPEC function $\left[c(\mathbf{r}) \geqslant 5.2 \times 10^{-3}\right]$ superimposed on the same image (for $S_{\mathrm{w}}=0.83$ ). The brown area has been invaded by the tracer and falls above the $c(\mathbf{r})$ threshold considered; the purple area has not been invaded by the tracer and falls below the $c(\mathbf{r})$ threshold; the red area has been invaded in the experiment but falls below the $c(\mathbf{r})$ threshold; and the blue area has not been invaded in the experiment but falls above the $c(\mathbf{r})$ threshold. (b) Variation of $\xi$ with $S_{\mathrm{w}}$. Comparison between the fraction of the wetting phase with a low eigenvector centrality value (gray region defined from centrality thresholds, $c(\mathbf{r}) \geqslant 4 \times 10^{-3}$ and $c(\mathbf{r}) \geqslant 5.2 \times 10^{-3}$ ) and the fraction of the wetting phase not involved in the solute transport process for the duration of the experiments (red circles) [26].

entropy and the eigenvector centrality to quantify the impact of water saturation on the entropy of the pore network: in this particular case, on the topology of the wetting phase (water), where solute transport is taking place. This entropy is defined as $H=-\sum_{i=1}^{n} p\left(c_{i}\right) \log p\left(c_{i}\right)$, where $p\left(c_{i}\right)$ is the probability of defining a node in the pore network with eigenvector centrality value $c_{i}$. The Shannon entropy results for the four saturations studied $\left[H\left(S_{\mathrm{w}}\right)\right]$ are $3.78(1.00)<4.02(0.83)<$ $4.03(0.77)<4.19(0.71)$. This demonstrates that, in general, entropy increases as saturation decreases. However, entropy is controlled not only by the bulk water saturation, but also by the distribution of the phases, as shown by the similar entropy values for saturations 0.83 and 0.77 .

In the case of transport, the concept of entropy has been widely used for thermodynamics studies in porous media [42], and less energy is destroyed as less entropy is generated (i.e., heat dissipation or conservation of temperature is slower). Its use for study of solute transport is less common (e.g., [43]). Different metrics have been proposed to describe and quantify solute mixing in porous media. The dilution index [44] quantifies dilution as the distribution of a solute mass over a porous medium volume, i.e., volume of the porous medium occupied by a solute cloud. This metric is expressed as the exponential of the Shannon entropy, computed from the solute concentration probability distribution. Using the eigenvector centrality probability distribution instead of the concentration could provide the "dilution potential" of the system. Therefore, the dilution potential, i.e., mixing capacity, increases as the water saturation decreases [26,36].

The pore-size distribution and pore connectivity are two of the main characteristics controlling fluid flow through porous media, i.e., the hydraulic conductivity or permeability of the system. However, in multiphase systems, and for the same porous medium, the permeability also depends on the bulk saturation of the phases and on its spatial distribution: the saturation-dependent permeability or relative permeability (e.g., $[45,46])$. In general, a decrease in permeability occurs as water saturation decreases (e.g., [26]). The eigenvector centrality measure can be used to infer the connectivity in a network (see Appendix A). Here, we define connectivity as $\Phi=\lambda_{N} / N$, where $\lambda_{N}$ is the maximum eigenvalue. Connectivity as calculated for the four saturations $\left[\Phi\left(S_{\mathrm{w}}\right)\right]$ is $1.34 \times 10^{-3}(1.00)>1.16 \times 10^{-3}(0.83)>$ $1.16 \times 10^{-3}(0.77)>1.14 \times 10^{-3}(0.71)$. This confirms that, in general, connectivity decreases as saturation decreases. Note that, as observed for entropy, similar connectivity values are obtained for saturations 0.83 and 0.77 , indicating that connectivity is also typically controlled by the distribution of the phases. While a different value of hydraulic conductivity for saturations 0.83 and 0.77 was obtained in laboratory experiments [26], the similar connectivity value for these two saturations shows that there are other topological controls on the hydraulic conductivity besides the connectivity.

Percolation theory, a mathematical framework that describes the properties of connected clusters in a random network [47], determines the macroscopic properties of a porous medium from its pore-scale connectivity statistics $[48,49]$. The percolation threshold $\rho_{c}$ is a critical probability below which the connections are too sparse to form a pathway through a sample, leading to an interrupted macroscopic flow and transport [35]. $\rho_{c}$ is nonuniversal and depends mainly on the pore connectivity and the wettability of the fluid to the solid surface. A global topological characteristic for an entire pore network is the coordination number $(z)$ - the number of pores connected to each other at each intersection, i.e., open to flow and transport. The most common case is $z=3$ and $z=6$ in $2 \mathrm{D}$ and $3 \mathrm{D}$ networks, respectively. Indeed, nodes with coordination numbers of 2 or less are very rare, while in highly connected networks we can have $z>6$.

The coordination value in percolation theory corresponds to the average node degree $\overline{\mathrm{deg}}$ as defined here. Karrer et al. [50] showed recently that $\rho_{c}$ is given by the inverse of the leading 
eigenvalue for the so-called nonbacktracking matrix [51]. The proposed expression is $\rho_{c}=1 /(\overline{\mathrm{deg}}-1)$. Although this definition of the percolation threshold was originally proposed for sparse networks (treelike networks), it is highly accurate for denser networks (many short loops) as well. The $\overline{\mathrm{deg}}$ value for the pore network defined in the fully saturated $\left(S_{\mathrm{w}}=1.00\right)$ experiment is 2.90 (see Table I). Hence $\rho_{c}$ for this porous medium is 0.526 , while the theoretical percolation threshold for 2D porous media is 0.590 [52].

Although the obtained percolation threshold is subject to uncertainty, it shows that the percolation threshold could be computed for any pore network from the proposed definition [51]. Note that the ability to determine the percolation threshold is relevant for immiscible displacement (i.e., drainage of one phase by imbibition of other) in the porous medium [52], as well as for solute transport $[35,53]$.

\section{CONCLUSION}

This work shows that network analysis and graph theory are extremely valuable for extraction of useful information about porous media and their transport properties. In particular, we have shown that the eigenvector centrality can be used to infer topological characteristics, controlling fluid flow and solute transport in porous media, as well as the impact on these characteristics when two immiscible phases are involved. By direct comparison with experiments we have shown that the proposed spatially projected eigenvector centrality function can give a reliable indication of the preferential zones through which fluids and solutes will potentially flow. In particular, this function can be used to infer the fraction of the porous medium that will behave as stagnation zones and/or preferential paths.

Properties such as the entropy of the network can be computed from the proposed method to give an idea of the intrinsic disorder of a particular porous medium or the disorder introduced by the presence of an immiscible phase. Note that entropy is a quantity that can be associated with measurable quantities such as tortuosity, but also with solute mixing, i.e., the "mixing capacity" of a system. The connectivity of porous media can also be analyzed by using the maximum eigenvalue of the adjacency matrix. Direct comparison with experiments also shows that there are other topological controls on the hydraulic conductivity besides the connectivity, highlighting one of the limitations of this technique.

We believe that the method introduced here can be viewed as a catalyst to promote research into the use of graph theory and network analysis to study, characterize, and predict dynamical flow and transport properties in porous media. This method could be used to characterize or parameterize porous and fractured media without solving discretized partial differential equations (e.g., Navier-Stokes), which are associated with high computational cost. Potential applications include in industry (filters, chemical reactors), applied energy (reservoirs), the environment (soils), and medicine (tissues).

\section{ACKNOWLEDGMENTS}

We acknowledge the Earth and Environmental Sciences and the Theoretical divisions of Los Alamos National Laboratory for fostering and supporting multidisciplinary collaborations. We express our gratitude to Dr. Yves Méheust and Dr. Tanguy Le Borgne (Geosciences Rennes, CNRS-University of Rennes 1, France) and Dr. Hervé Tabuteau (Physics Institute of Rennes, CNRS-University of Rennes 1, France), who were involved in the millifluidic transport experiments. We are thankful to Dr. Gabriela Galassi for valuable discussions on statistics.

Both authors contributed equally to this work. (a)

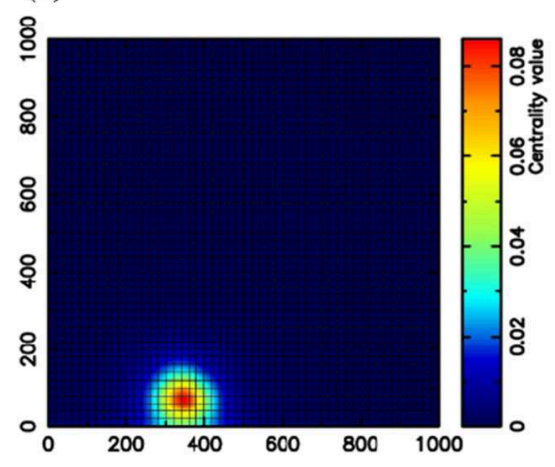

(c)

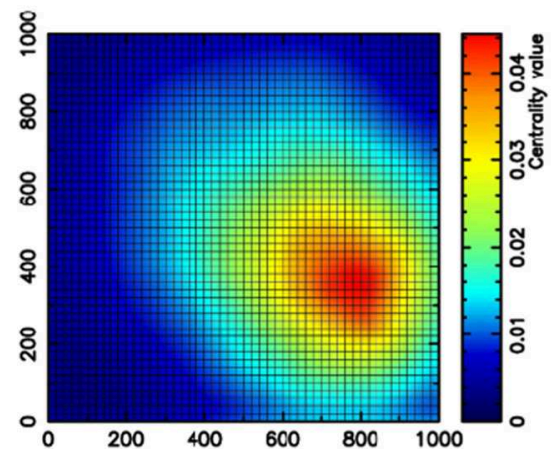

(b)

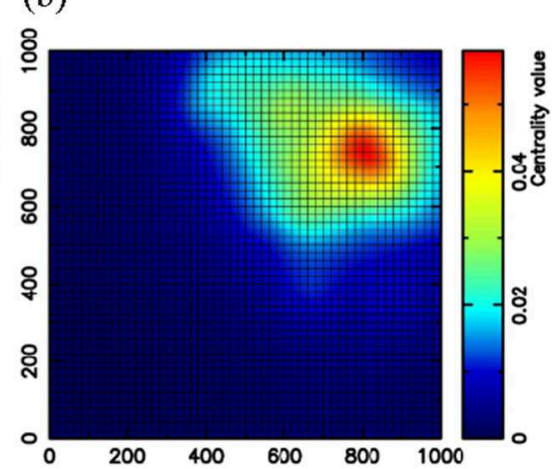

(d)

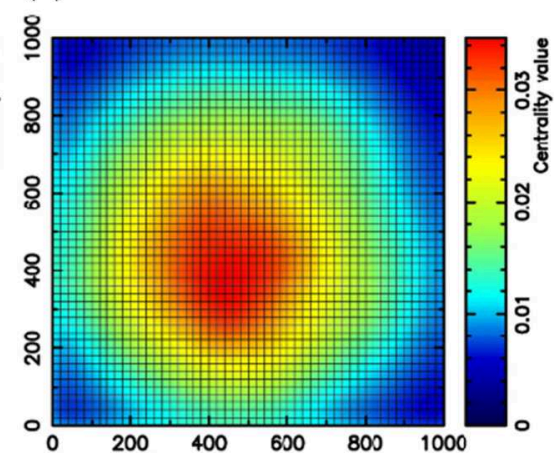

FIG. 5. Different degrees of connectivity. Connectivity is limited to (a) first, (b) second, (c) third, and (d) fourth nearest neighbors. Weights $w_{i j}$ are chosen at random between 0.5 and 1.0. 


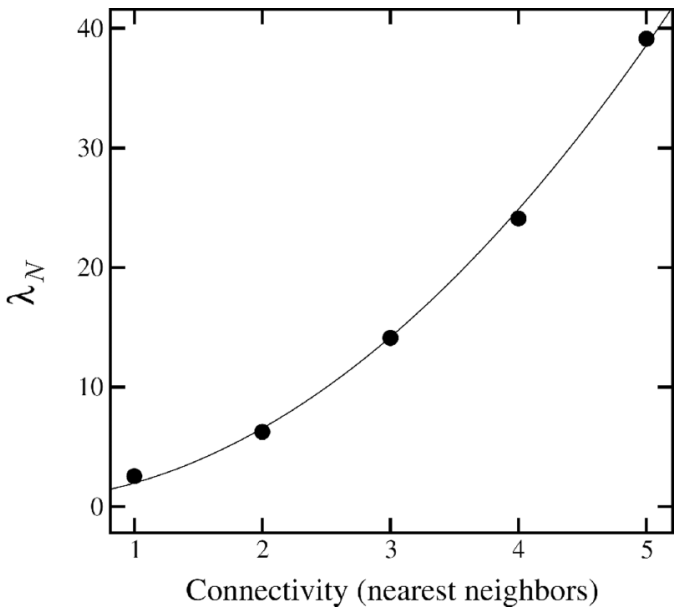

FIG. 6. $\lambda_{N}$ as a function of the lattice connectivity (number of neighbors).

\section{APPENDIX A: EIGENVECTOR CENTRALITY LOCALIZATION PROBLEM}

In this example, we show how the localization changes with the connectivity for a regular network. The more connected the nodes are among each other, the less the centrality is localized. For only first-nearest-neighbor connectivity, the centrality shows a highly localized hub, which is depicted as the small red area in Fig. 5(a). As the connectivity is increased beyond the first nearest neighbors, the localization is slowly removed (the hub spreads across a larger region).

\section{Relationship between $\lambda_{N}$ and connectivity}

The degree of connectivity can be estimated with the maximum eigenvalue $\lambda_{N}$. In Fig. 6 , we show how $\lambda_{N}$ changes with connectivity. The quadratic behavior is due to the fact that in this particular case, $\lambda_{N}$ accounts for the average degree. As connectivity is increased from first to higher nearest neighbors, the average degree increases in a quadratic fashion. For this particular case of regular lattices, $\lambda_{N}$ shows a linear behavior as a function of the average degree.

\section{Localization and randomization of edge weights}

Figure 7 shows that when the edge weights $w_{i j}$ are chosen at random, localization occurs. For a regular lattice with equal weights, the localization is removed.

\section{APPENDIX B: EFFECT OF APPLYING THE CORRECTION $\delta$}

We can transition from very localized to delocalized centrality values, for low and large values of "spurious" connectivity $\delta$, respectively, as shown in Fig. 8.

After applying the correction $\delta$, the effect on $\lambda$ (see the text) is that the largest eigenvalue $\lambda_{N}$ separates from the second largest eigenvalue (see Fig. 9).

\section{APPENDIX C: WEIGHT DISTRIBUTION OF A PORE-NETWORK WEIGHTED GRAPH}

The weights for a physical network, as the cases studied here (pore network), show a Gaussian type of distribution with a peak around a very well-defined medium value. In Fig. 10, weights $w_{i j}=\exp \left(-s_{0} / s_{i j}\right)$ from the network with 0.83 water saturation $\left(S_{\mathrm{w}}\right)$ have been computed. $s_{i j}$ is the 2D effective cross section (pore throat) that connects node $i$ with node $j$. $s_{0}$ is a normalization coefficient chosen as 1 pixel unit.

\section{APPENDIX D: NOTES ON THE TWO-DIMENSIONAL LORENTZIAN DENSITY FUNCTION}

Note that the Lorentzian probability function defined for $\mathbb{R}^{2}$ cannot be normalized in its whole domain [33]. In other words, the integral

$$
\iint_{\mathrm{D}} \mathcal{L}\left(\mathbf{r}-\mathbf{r}_{i}\right) d s=\int_{-\infty}^{\infty} \int_{-\infty}^{\infty} \mathcal{L}\left((x, y)-\left(x_{i}, y_{i}\right)\right) d y d x
$$

is not convergent. For the purpose of this work, we consider that the Lorentzian probability function is defined only for a radial extension limited by a cutoff radius $r_{\text {cut }}$. Hence,

$$
\int_{0}^{2 \pi} \int_{0}^{r_{\mathrm{cut}}} \frac{\zeta}{2 \pi} \frac{\Gamma}{r^{2}+(\Gamma / 2)^{2}} r d r d \theta=1
$$

(a)

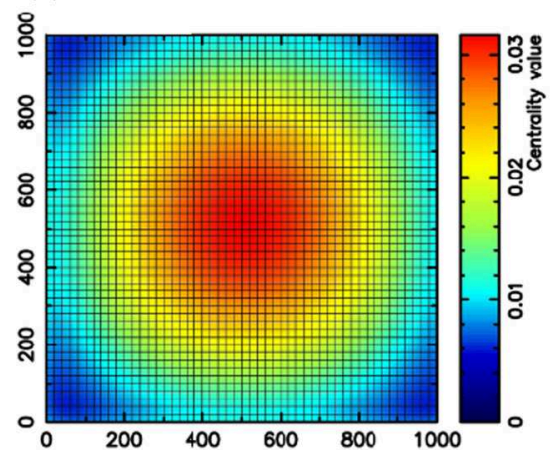

(b)

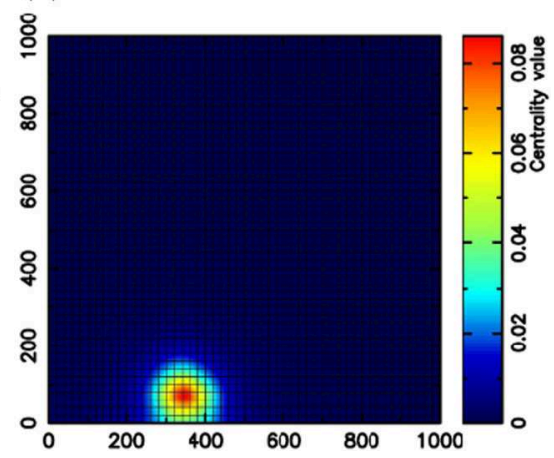

FIG. 7. Comparison between (a) a pristine firstneighbor-connected lattice and (b) a regular graph with randomized weights where $0.5 \leqslant w_{i j} \leqslant 1.0$. 
(a)

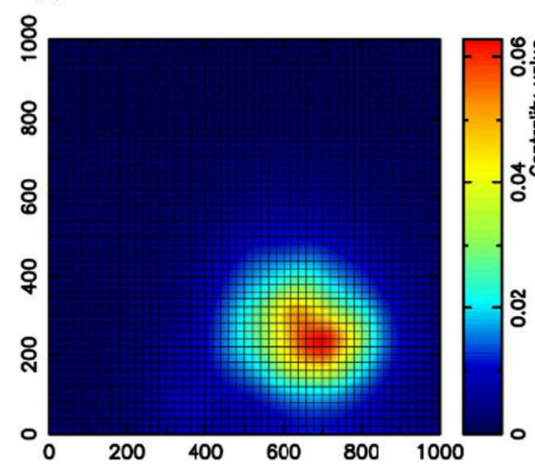

(b)

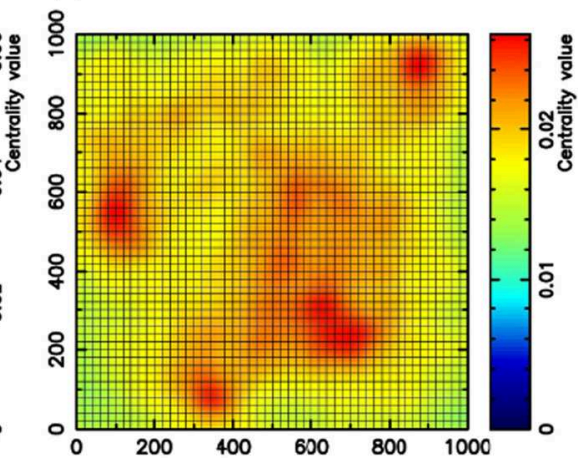

FIG. 8. (a) Original compared to (b) corrected weights for a network where $0.5 \leqslant w_{i j} \leqslant 1.0$ and with $\delta=10^{-4}$. where $r$ and $\theta$ are the the vector norm $\left(\left\|\mathbf{r}-\mathbf{r}_{i}\right\|\right)$ and the polar angle, respectively. Solving for $\zeta$, we have

$$
\zeta=\frac{-2}{\Gamma\left(2 \ln (\Gamma)-\ln \left(4\left(r_{\mathrm{cut}}\right)^{2}+\Gamma^{2}\right)\right)}
$$

\section{APPENDIX E: PROBABILISTIC INTERPRETATION OF THE EIGENVECTOR CENTRALITY}

As mentioned in the text, $\iint_{\mathrm{D}} \rho(\mathbf{r}) d s=N$ for a restricted domain $\mathrm{D}$ (network region). Moreover, $p(\mathbf{r})=\rho(\mathbf{r}) / N$ can be viewed as a probability density function such that $\langle c\rangle=$ $\iint_{\mathrm{D}} c(\mathbf{r}) p(\mathbf{r}) d s$.

Note that the "square" of a centrality value $\left(c_{i}^{2}\right)$ can be viewed as the conditional probability of finding a particle flowing through a node $i$, hence, the function $p_{p}(\mathbf{r}) \equiv c^{2}(\mathbf{r}) p(\mathbf{r})$ can be interpreted as the local probability density function for finding "any particle" flowing at point $\mathbf{r}$. On the other hand, $\left\langle c^{2}\right\rangle=\iint_{\mathrm{D}} c^{2}(\mathbf{r}) p(\mathbf{r}) d s=1 / N$, given that $\sum_{i}^{N} c_{i}^{2}=1$.

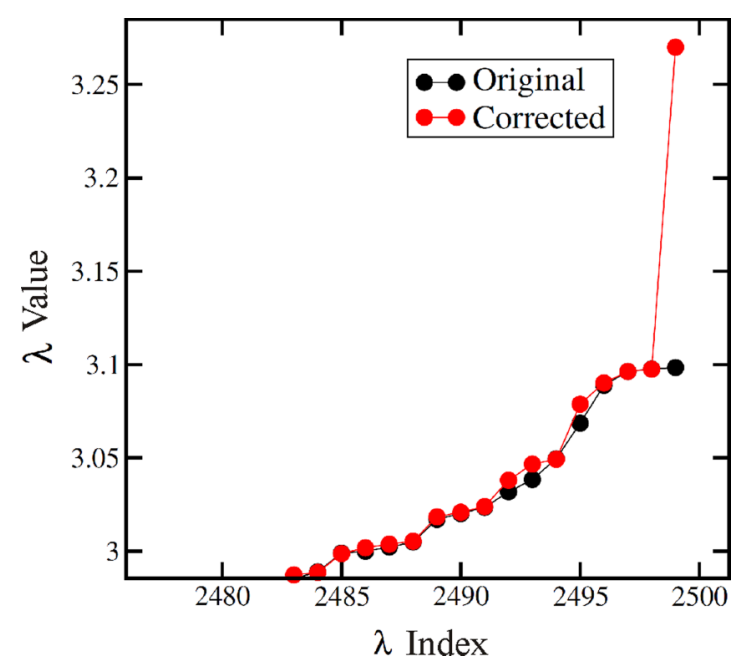

FIG. 9. Correction of the centrality where $0.5 \leqslant w_{i j} \leqslant 1.0$ and with $\delta=10^{-4}$
The local probability density function $p_{p}(\mathbf{r})$ can be written as follows:

$$
p_{p}(\mathbf{r})=\sum_{i}^{N} c_{i}^{2} \mathcal{L}\left(\mathbf{r}-\mathbf{r}_{i}\right)
$$

In summary, we have the following.

(i) $\mathcal{L}\left(\mathbf{r}-\mathbf{r}_{i}\right)$ : probability of finding "nodei" at point $\mathbf{r}$. See Fig. 11.

(ii) $p(\mathbf{r})=\rho(\mathbf{r}) / N$ : probability of finding "any node" at point $\mathbf{r}$.

(iii) $c_{i}^{2}$ : conditional probability of finding a particle flowing through node $i$ (or given that the position is $\mathbf{r}_{i}$ ).

(iv) $p_{p}(\mathbf{r})$ : probability of finding "a particle flowing" at position $\mathbf{r}$.

Result 1 . The probability of finding node $i$ has a Lorentzian type of distribution.

The uncertainty in $d_{i j}$ (distance between nodes) will determine the uncertainty of $\mathbf{r}_{i}$ if we assume that all the rest of the connected nodes are in an exact position $\mathbf{r}_{j \neq i}$. We

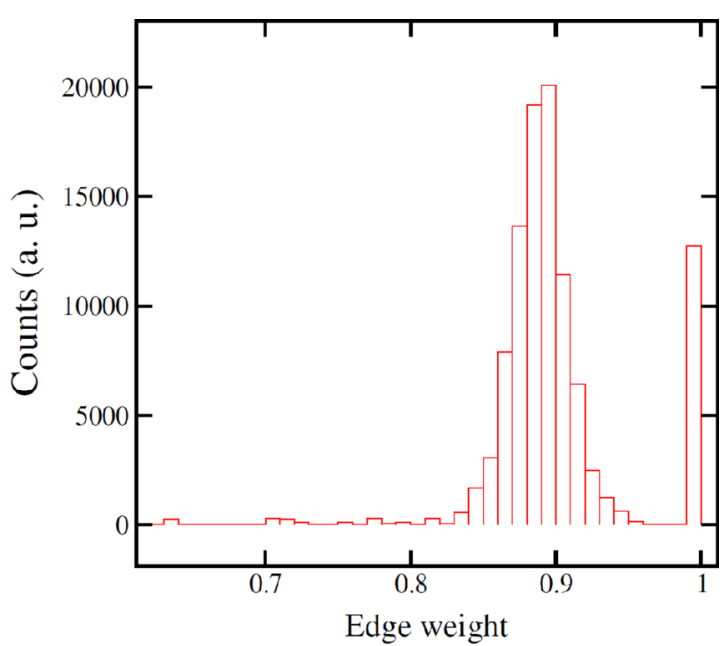

FIG. 10. Histogram of edge weights $w_{i j}$ computed for the pore network with $S_{\mathrm{w}}=0.83$ (see the text). A medium value of $w \simeq 0.9$ is shown. 


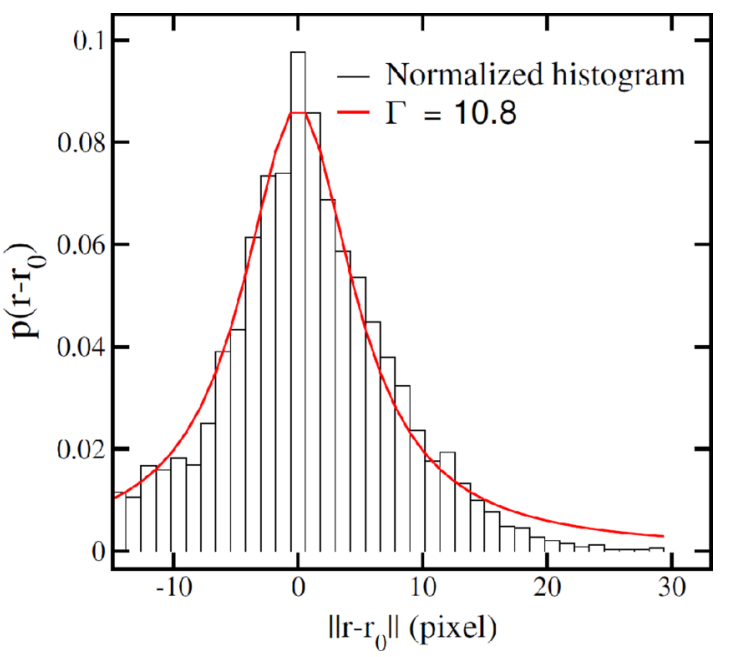

FIG. 11. Lorentzian fit of a normalized histogram of the deviation of node position. The Lorentzian (red curve) with $\Gamma=10.8$ adjusts very well to the normalized histogram. This distribution can be seen as the probability of finding node $i$ at position $\mathbf{r}_{i}$.

hence proceed by collecting all the distances $d_{i j}$, where $j$ is a neighbor of $i$, and use this distribution to approximate the distribution of a general position $\mathbf{r}$ around a node in $\mathbf{r}_{0}$.

Result 2. $p_{p}(\mathbf{r})$ is a probability density function.

We begin to verify that $\iint_{\mathrm{D}} p_{p}(\mathbf{r}) d s=1$ :

$$
\begin{gathered}
\iint_{\mathrm{D}} p_{p}(\mathbf{r}) d s=\iint_{\mathrm{D}} \sum_{i}^{N} c_{i}^{2} \mathcal{L}\left(\mathbf{r}-\mathbf{r}_{i}\right) d s, \\
\iint_{\mathrm{D}} p_{p}(\mathbf{r}) d s=\sum_{i}^{N} c_{i}^{2} \iint_{\mathrm{D}} \mathcal{L}\left(\mathbf{r}-\mathbf{r}_{i}\right) d s, \\
\iint_{\mathrm{D}} p_{p}(\mathbf{r}) d s=\sum_{i}^{N} c_{i}^{2} \iint_{\mathrm{D}} \mathcal{L}\left(\mathbf{r}-\mathbf{r}_{i}\right) d s=\sum_{i}^{N} c_{i}^{2}=1 .
\end{gathered}
$$

Note that $p_{p}(\mathbf{r})=\sum_{i}^{N} c_{i}^{2} \mathcal{L}\left(\mathbf{r}-\mathbf{r}_{i}\right) \geqslant 0$, since $c_{i}^{2}>0 \forall i$ and $\mathcal{L}\left(\mathbf{r}-\mathbf{r}_{i}\right)>0 \forall i \forall \mathbf{r}$.

(a)

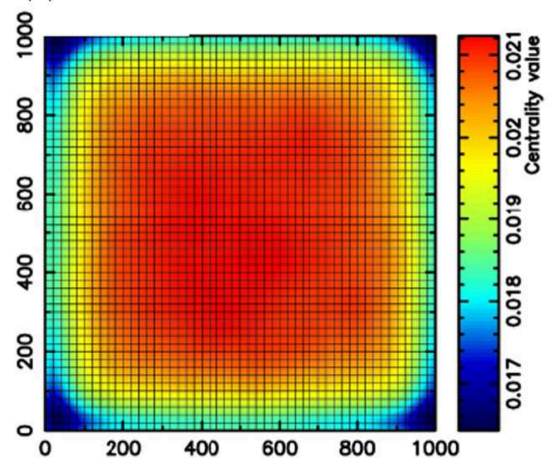

(b)

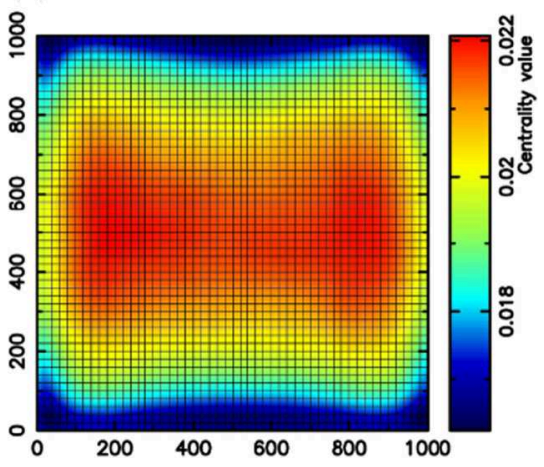

Result 3. $\left\langle c^{2}\right\rangle=\iint_{\mathrm{D}} c^{2}(\mathbf{r}) p(\mathbf{r}) d s=1 / N$.

Projection $c^{2}(\mathbf{r})$ is defined using the node density as follows:

$$
c^{2}(\mathbf{r})=\frac{1}{\rho(\mathbf{r})} \sum_{i}^{N} c_{i}^{2} \mathcal{L}\left(\mathbf{r}-\mathbf{r}_{i}\right) .
$$

Hence,

$$
\begin{gathered}
\iint_{\mathrm{D}} c^{2}(\mathbf{r}) p(\mathbf{r}) d s=\iint_{\mathrm{D}} \frac{1}{\rho(\mathbf{r})} \sum_{i}^{N} c_{i}^{2} \mathcal{L}\left(\mathbf{r}-\mathbf{r}_{i}\right) \frac{\rho(\mathbf{r})}{N} d s, \\
\iint_{\mathrm{D}} c^{2}(\mathbf{r}) p(\mathbf{r}) d s=\frac{1}{N} \sum_{i}^{N} c_{i}^{2} \iint_{\mathrm{D}} \mathcal{L}\left(\mathbf{r}-\mathbf{r}_{i}\right) d s \\
=\frac{1}{N} \sum_{i}^{N} c_{i}^{2}=\frac{1}{N}
\end{gathered}
$$

\section{APPENDIX F: EIGENVECTOR CENTRALITY CONDITIONED BY THE MEAN FLOW DIRECTION}

Figure 12 shows the effect of the centrality values when the adjacency matrix is conditioned by the mean flow direction. We can observe that when the bias is applied along the $x$ direction, the upper or lower limits of the network become less conductive (less central) compared to the unbiased case.

\section{APPENDIX G: SPEC FUNCTION COMPARED TO CENTRALITY DOTTED PLOT (FIG. 13)}

\section{APPENDIX H: ESTIMATION OF THE VARIABILITY OF THE STATISTICAL FUNCTION COMPUTED FROM THE CENTRALITY DISTRIBUTION}

Implementing the bootstrap method, we estimate the variability of each statistical function, mean $(\mu)$, standard deviation $(\sigma)$, and skewness $(\gamma)$, and for each saturation degree. Details about the bootstrap method can be found in [54] and [55]. The results from the implementation of the bootstrap method were corroborated with the estimation of the standard error of $\mu$ using the central limit theorem, which is less preferred than the bootstrap because in this case the assumption of independent and identically distributed (i.i.d.) random
FIG. 12. (a) Unbiased and (b) $x$-biased spatially projected eigenvector centrality (SPEC) function for a random weighted graph where $0.5 \leqslant w_{i j} \leqslant 1.0$ and with $\delta=10^{-4}$. 
(a)

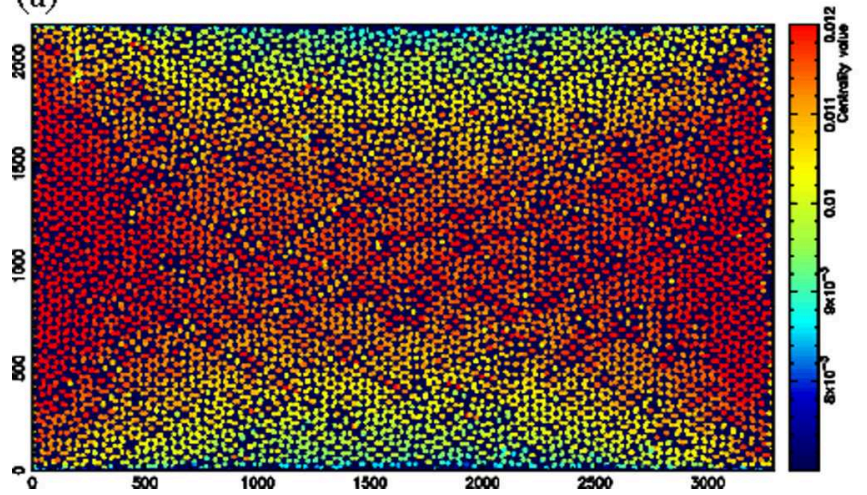

(b)

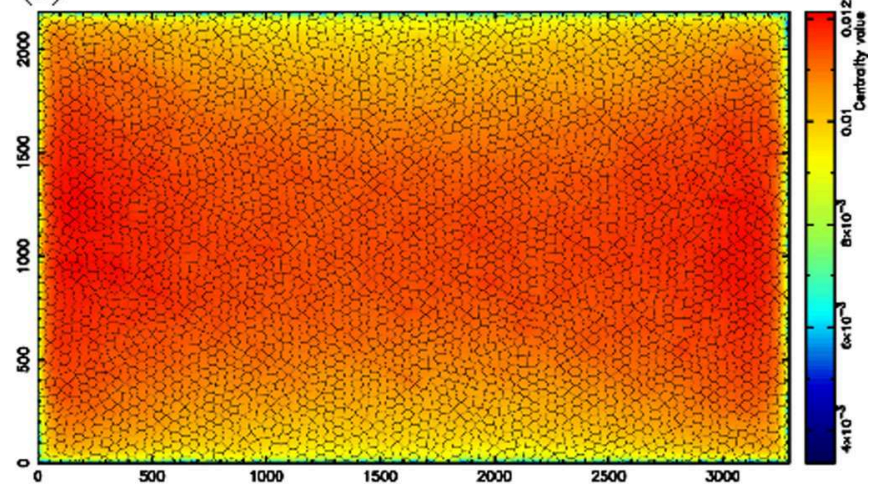

FIG. 13. (a) Centrality measure of each node of the pore network and (b) spatially projected eigenvector centrality (SPEC) function for the saturation degree $S_{\mathrm{w}}=1.00$. Note that the scales are different because the SPEC function smooths out very high and low values.

variables is not fulfilled. The errors in the statistical functions are listed in Table III (updated from Table II in the text).

Significant differences in $\mu_{c_{i}}$ are observed between all saturation degrees. In the case of $\sigma_{c_{i}}$, saturations 0.83 and 0.71 can be considered equal. Finally, $\gamma_{c_{i}}$ can be considered equal for all saturation degrees.

\section{APPENDIX I: PSEUDOCODE TO COMPUTE THE SPATIALLY PROJECTED EIGENVECTOR CENTRALITY FUNCTION}

In this pseudocode, the adjacency matrix $A$ is diagonalized and the principal eigenvector $\mathbf{c}$ is projected onto $\mathbf{r}$ using the node density $\rho(\mathbf{r})$. Here, the $x$ and $y$ components of $\mathbf{r}$ are discretized in $M$ points of step $d x$ and $d y$, respectively. $c_{k}$ and $\mathbf{r}_{k}$ are the centrality and position for every node $k . N$ is the total number of nodes. $\lambda_{N}$ is the maximum eigenvalue of matrix $A$, and $U$ is the matrix containing all the eigenvectors. $\mathcal{L}\left(\mathbf{r}-\mathbf{r}_{k}\right)$ is the Lorentzian function, with parameter $\Gamma$, applied to the $\mathbf{r}-\mathbf{r}_{k}$ variable.

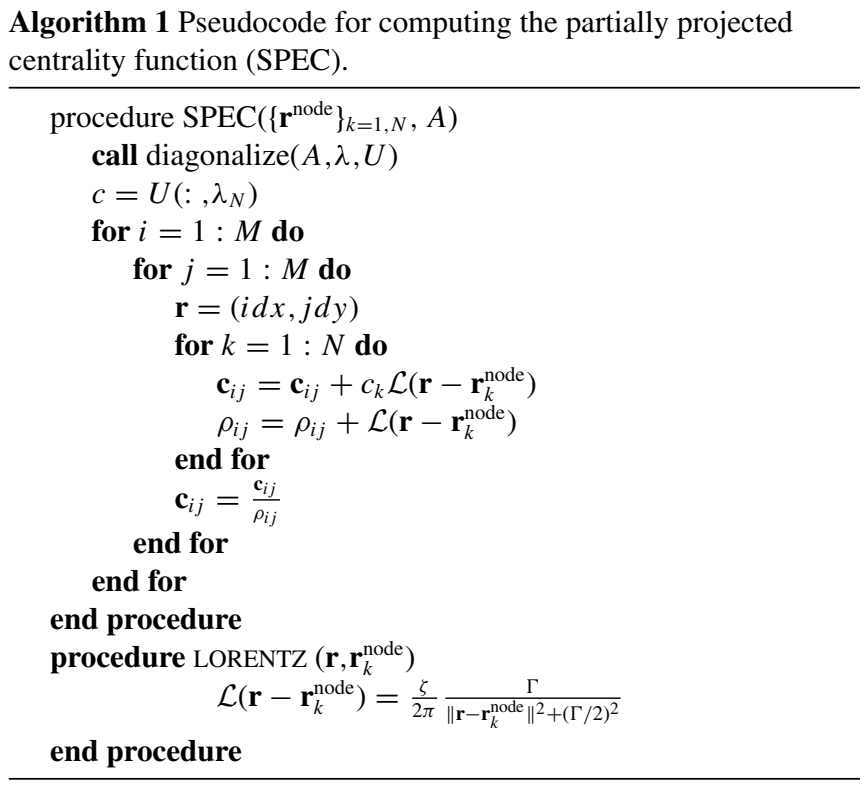

TABLE III. Moments of the eigenvector centrality distribution, including the mean $\mu_{c_{i}}$, standard deviation $\sigma_{c_{i}}$, and skewness $\gamma_{c_{i}}$, for different water saturation $S_{\mathrm{w}}$ values: $1.00,0.83,0.77$, and 0.71 . Errors were estimated from the bootstrap method. $N$ is the total number of nodes for each case.

\begin{tabular}{ccccccc}
\hline \hline$S_{\mathrm{w}}$ & $\mu_{c_{i}}$ & Error & $\mu_{c_{i}} / N$ & $\sigma_{c_{i}}$ & Error & $\gamma_{c_{i}}$ \\
\hline 1.00 & $1.12 \times 10^{-2}$ & $1.02 \times 10^{-5}$ & $1.03 \times 10^{-5}$ & $9.06 \times 10^{-4}$ & $7.72 \times 10^{-6}$ & -0.31 \\
0.83 & $9.30 \times 10^{-3}$ & $8.44 \times 10^{-6}$ & $8.86 \times 10^{-6}$ & $7.28 \times 10^{-4}$ & $5.78 \times 10^{-6}$ & -0.32 \\
0.77 & $9.50 \times 10^{-3}$ & $9.04 \times 10^{-6}$ & $8.81 \times 10^{-6}$ & $7.62 \times 10^{-4}$ & $6.26 \times 10^{-6}$ & -0.30 \\
0.71 & $9.10 \times 10^{-4}$ & $8.85 \times 10^{-6}$ & $8.73 \times 10^{-6}$ & $7.27 \times 10^{-4}$ & $5.91 \times 10^{-6}$ & -0.26 \\
\hline \hline
\end{tabular}


[1] J. Bear, Dynamics of Fluids in Porous Media (American Elsevier, New York, 1972).

[2] K. A. Rejniak, V. Estrella, T. Chen, A. S. Cohen, M. Lloyd, and D. L. Morse, Front. Oncol. 3, 111 (2013).

[3] J. Valdes-Abellan, J. Jiménez-Martínez, L. Candela, D. Jacques, C. Kohfahl, and K. Tamoh, J. Hydrol. 549, 114 (2017).

[4] R. S. Middleton, J. W. Carey, R. P. Currier, J. D. Hyman, Q. Kang, S. Karra, J. Jiménez-Martínez, M. L. Porter, and H. S. Viswanathan, Appl. Energy 147, 500 (2015).

[5] M. M. Britton, A. J. Sederman, A. F. Taylor, S. K. Scott, and L. F. Gladden, J. Phys. Chem. A 109, 8306 (2005).

[6] S. C. Cowin and L. Cardoso, J. Biomech. 48, 842 (2015).

[7] I. Fatt, Petrol. Trans., AIME 207, 144 (1956).

[8] H. Takase, K. J. Worgan, M. D. Impey, and P. Grindrod, SIAM J. Appl. Math. 57, 597 (1997).

[9] M. A. Celia, P. C. Reeves, and L. A. Ferrand, Rev. Geophys. 33, 1049 (1995).

[10] M. J. Blunt, Curr. Opin. Colloid Interface Sci. 6, 197 (2001).

[11] P. Bonacich, Am. J. Sociol. 92, 1170 (1987).

[12] T. Opsahl, F. Agneessens, and J. Skvoretz, Soc. Netw. 32, 245 (2010).

[13] Y. W. Tsang and C. F. Tsang, Water Resour. Res. 25, 2076 (1989).

[14] L. Moreno and C. F. Tsang, Water Resour. Res. 30, 1421 (1994).

[15] P. Grindrod and M. Impey, Water Resour. Res. 29, 4077 (1993).

[16] O. A. Cirpka, G. Chiogna, M. Rolle, and A. Bellin, Water Resour. Res. 51, 241 (2015).

[17] D. Russo, Water Resour. Res. 31, 1647 (1995).

[18] D. Russo, A. Hadad, and A. Laufer, Adv. Water Resour. 21, 63 (1998).

[19] A. De Wit, Phys. Rev. Lett. 87, 054502 (2001).

[20] C. D. Meyer, Matrix Analysis and Applied Linear Algebra (SIAM, Philadelphia, 2009), Vol. 2.

[21] T. Martin, X. Zhang, and M. E. J. Newman, Phys. Rev. E 90, 052808 (2014).

[22] E. Abrahams, P. W. Anderson, D. C. Licciardello, and T. V. Ramakrishnan, Phys. Rev. Lett. 42, 673 (1979).

[23] P. Lawrence, B. Sergey, R. Motwani, and T. Winograd, Technical Report, Stanford University (1998).

[24] S. Brin and L. Page, Comput. Netw. ISDN Syst. 30, 107 (1998).

[25] M. A. Rodriguez, Knowl.-Based Syst. 21, 727 (2008).

[26] J. Jiménez-Martínez, T. Le Borgne, H. Tabuteau, and Y. Méheust, Water Resour. Res. 53, 1457 (2017).

[27] T. Y. Kong and A. Rosenfeld, Topological Algorithms for Digital Image Processing (Elsevier, Amsterdam, 1996), Vol. 19.

[28] C. R. Maurer, R. Qi, and V. Raghavan, IEEE Trans. Pattern Anal. Mach. Intel. 25, 265 (2003).

[29] W. B. Lindquist, S.-M. Lee, D. A. Coker, K. W. Jones, and P. Spanne, J. Geophys. Res. B 101, 8297 (1996).
[30] R. Kimmel, D. Shaked, N. Kiryati, and A. M. Bruckstein, Comput. Vis. Image Und. 62, 382 (1995).

[31] W. C. Sun, J. E. Andrade, and J. W. Rudnicki, Int. J. Num. Meth. Eng. 88, 1260 (2011).

[32] B. Aradi, N. Bock, S. M. Mniszewski, J. Mohd-Yusof, and C. F. A. Negre, https://qmmd.github.io.

[33] L. Filipovic and S. Selberherr, Proceedings of the 8th IMACS Seminar on Monte Carlo Methods and Applications (De Gruyter, Berlin, 2013).

[34] E. Aker, K. J. Måløy, A. Hansen, and G. G. Batrouni, Transp. Porous Med. 32, 163 (1998).

[35] P. G. d. Gennes, J. Fluid Mech. 136, 189 (1983).

[36] J. Jiménez-Martínez, P. de Anna, H. Tabuteau, R. Turuban, T. Le Borgne, and Y. Méheust, Geophys. Res. Lett. 42, 5316 (2015).

[37] G. Taylor, Philos. Trans. Roy. Soc. A 219, 186 (1953).

[38] D. Bolster, M. Dentz, and T. Le Borgne, Phys. Fluids 21, 056601 (2009).

[39] J. Bouquain, Y. Méheust, D. Bolster, and P. Davy, Phys. Fluids 24, 083602 (2012).

[40] M. Dehmer and A. Mowshowitz, Info. Sci. 181, 57 (2011).

[41] A. Lesne, Math. Struct. Comput. Sci. 24, e240311 (2014).

[42] M. Torabi, K. Zhang, G. Yang, J. Wang, and P. Wu, Energy 82, 922 (2015).

[43] G. Chiogna, D. L. Hochstetler, A. Bellin, P. K. Kitanidis, and M. Rolle, Geophys. Res. Lett. 39 (2012).

[44] P. K. Kitanidis, Water Resour. Res. 30, 2011 (1994).

[45] R. H. Brooks and A. T. Corey, Hydraulic Properties of Porous Media, Hydrology Papers, No. 3 (Colorado State University, Fort Collins, 1964).

[46] M. Th. van Genuchten, Soil Sci. Soc. Am. J. 44, 892 (1980).

[47] S. R. Broadbent and J. M. Hammersley, in Math. Proc. Cambr. Philos. Soc. (Cambridge University Press, Cambridge, UK, 1957), Vol. 53, pp. 629-641.

[48] M. Sahimi, Flow and Transport in Porous Media and Fractured Rock: From Classical Methods to Modern Approaches (John Wiley \& Sons, New York, 2011).

[49] B. Ghanbarian, H. Daigle, A. G. Hunt, R. P. Ewing, and M. Sahimi, J. Geophys. Res. B 120, 182 (2015).

[50] B. Karrer, M. E. J. Newman, and L. Zdeborová, Phys. Rev. Lett. 113, 208702 (2014).

[51] F. Krzakala, C. Moore, E. Mossel, J. Neeman, A. Sly, L. Zdeborová, and P. Zhang, Proc. Natl. Acad. Sci. USA 110, 20935 (2013).

[52] B. Berkowitz and I. Balberg, Water Resour. Res. 29, 775 (1993).

[53] T. Le Borgne, M. Dentz, and E. Villermaux, J. Fluid Mech. 770, 458 (2015).

[54] B. Efron, in Bootstrap Methods: Another Look at the Jackknife (Springer, New York, 1992), pp. 569-593.

[55] B. Efron and R. J. Tibshirani, An Introduction to the Bootstrap (CRC Press, Boca Raton, FL, 1994). 